\title{
DISCOVERY OF A BROWN DWARF COMPANION TO GLIESE 570ABC: A 2MASS T DWARF SIGNIFICANTLY COOLER THAN GLIESE 229B
}

\author{
Adam J. Burgasser, ${ }^{1}$ J. Davy Kirkpatrick, ${ }^{2}$ Roc M. Cutri,${ }^{2}$ Howard McCallon, ${ }^{2}$ Gene Kopan, ${ }^{2}$ \\ John E. Gizis, ${ }^{3}$ James Liebert, ${ }^{4}$ I. Neill Reid,${ }^{5}$ Michael E. Brown,${ }^{6,7}$ David G. Monet,${ }^{8}$ \\ Conard C. Dahn ${ }^{8}$ Charles A. Beichman, ${ }^{9}$ And Michael F. Skrutskie ${ }^{3}$ \\ Received 1999 November 22; accepted 2000 January 11; published 2000 February 10
}

\begin{abstract}
We report the discovery of a widely separated $(258.3 \pm 0$ "..4) T dwarf companion to the Gl 570ABC system. This new component, Gl 570D, was initially identified from the Two Micron All-Sky Survey. Its near-infrared spectrum shows the 1.6 and $2.2 \mu \mathrm{m} \mathrm{CH}_{4}$ absorption bands characteristic of $\mathrm{T}$ dwarfs, while its common proper motion with the Gl 570ABC system confirms companionship. Gl 570D $\left(M_{J}=16.47 \pm 0.07\right)$ is nearly a full magnitude dimmer than the only other known T dwarf companion, Gl 229B, and estimates of $L=(2.8 \pm$ $0.3) \times 10^{-6} L_{\odot}$ and $T_{\text {eff }}=750 \pm 50 \mathrm{~K}$ make it significantly cooler and less luminous than any other known brown dwarf companion. Using evolutionary models by Burrows et al. and an adopted age of 2-10 Gyr, we derive a mass estimate of $50 \pm 20 M_{\text {Jup }}$ for this object.
\end{abstract}

Subject headings: binaries: visual — infrared: stars — stars: fundamental parameters stars: individual (Gliese 570D) — stars: low-mass, brown dwarfs

\section{INTRODUCTION}

Direct detection techniques, like those used in the discovery of the prototype T dwarf Gl 229B (Nakajima et al. 1995; Oppenheimer 1999), have been used for the last 15 years to search for brown dwarfs around nearby stars. ${ }^{10}$ Despite the large samples involved, only two bona fide brown dwarf companions have been directly detected, Gl 229B and the young L-type brown dwarf G196-3B (Rebolo et al. 1998). ${ }^{11}$ Since most of these searches have been confined to a narrow field of view around the primary (typically $10-60^{\prime \prime}$ ), widely separated companions $^{12}$ may be missed. Indeed, both G196-3B and Gl 229B are less than $20^{\prime \prime}$ from their primary. Field surveys, such as the Two Micron All-Sky Survey (2MASS; Skrutskie et al. 1997), the Deep Near Infrared Survey (Epchtein et al. 1997), and the Sloan Digital Sky Survey (SDSS; Gunn \& Weinberg 1995), overcome this limitation. Indeed, J. D. Kirkpatrick et al. (2000, in preparation) have recently identified two L-type brown dwarf companions at wide separation.

We are currently searching the 2MASS catalogs for field T dwarfs (Burgasser et al. 1998), brown dwarfs spectrally identified by $\mathrm{CH}_{4}$ absorption bands at 1.6 and $2.2 \mu \mathrm{m}$ (Kirkpatrick et al. 1999b). One of our discoveries, 2MASSW

\footnotetext{
${ }^{1}$ Division of Physics, M/S 103-33, California Institute of Technology, Pasadena, CA 91125; diver@its.caltech.edu.

${ }^{2}$ Infrared Processing and Analysis Center, M/S 100-22, California Institute of Technology, Pasadena, CA 91125.

${ }^{3}$ Five College Astronomy Department, Department of Physics and Astronomy, University of Massachusetts, Amherst, MA 01003.

${ }^{4}$ Steward Observatory, University of Arizona, Tucson, AZ 85721.

${ }^{5}$ Department of Physics and Astronomy, University of Pennsylvania, 209 South 33d Street, Philadelphia, PA 19104-6396.

${ }^{6}$ Division of Geological and Planetary Sciences, M/S 105-21, California Institute of Technology, Pasadena, California 91125.

${ }^{7}$ Alfred P. Sloan Research Fellow.

${ }^{8}$ US Naval Observatory, P.O. Box 1149, Flagstaff, AZ 86002.

${ }^{9}$ Jet Propulsion Laboratory, M/S 180-703, 4800 Oak Grove Drive, Pasadena, CA 91109.

${ }^{10}$ For a review of these companion searches, see Oppenheimer (1999).

${ }^{11}$ The companion object GD 165B (Becklin \& Zuckerman 1989) may also be a brown dwarf, although its status is questionable (Kirkpatrick et al. 1999a).

${ }^{12}$ We adopt an observational definition for "widely separated" as angular separation greater than 100"; see Fischer \& Marcy (1992).
}

J1457150-212148 (hereafter Gl 570D), has been confirmed as a widely separated, common proper-motion companion to the Gl 570ABC system. This system is comprised of a $\mathrm{K} 4 \mathrm{~V}$ primary and a M1.5 V-M3 V close binary (Duquennoy \& Mayor 1988; Mariotti et al. 1990; Forveille et al. 1999) at a distance of $5.91 \pm 0.06 \mathrm{pc}$ (Perryman et al. 1997). In $\S 2$ we describe the selection of this object from the 2MASS database, review subsequent observations, and establish its common proper motion with Gl 570ABC. In $\S 3$ we estimate $L$ and $T_{\text {eff }}$ of Gl 570D based on its distance and brightness and make $T_{\text {eff }}$ and mass estimates using the evolutionary models of Burrows et al. (1997).

\section{IDENTIFICATION OF G1 570D}

\subsection{Selection and Confirmation of Gl 570D}

Gl 570D was initially selected as a $\mathrm{T}$ dwarf candidate from the 2MASS Point Source Catalog. $\mathrm{T}$ dwarf candidates were required to have $J$ - and $H$-band detections with $J<16$ (2MASS signal-to-noise ratio $\sim 10$ limit), $J-H<0.3$ and $H-K_{s}<0.3$ (limit or detection), $|b|>15^{\circ}$ (to eliminate source confusion in the Galactic plane), and no optical counterpart in the USNOA catalog (Monet et al. 1998) within 10". Close optical doubles not identified by USNO-A and proper-motion stars were eliminated by examination of Digitized Sky Survey (DSS) images of the SERC-J and AAO SES (Morgan et al. 1992) surveys. Our search criteria are also sensitive to minor planets, due to their intrinsically blue near-infrared colors (Veeder et al. 1995; Sykes et al. 1999), lack of optical counterpart at an earlier epoch, and pointlike appearance due to the short 2MASS exposure time $(7.8 \mathrm{~s})$. Follow-up near-infrared imaging to eliminate these objects from our candidate pool was carried out on the Cerro Tololo Infrared Imager on the Cerro Tololo InterAmerican Observatory (CTIO) Ritchey-Chretien $1.5 \mathrm{~m}$ telescope during 1999 July 23-25 (UT). Gl 570D was one of only 11 candidates detected in these observations (the remaining candidates were likely asteroids). Optical images of the Gl 570D field from the SERC-J and AAO SES surveys, as well as 2MASS $J$ - and $K_{s}$-band images, are shown in Figure 1; the Gl 570ABC triple can be seen in the lower left-hand 

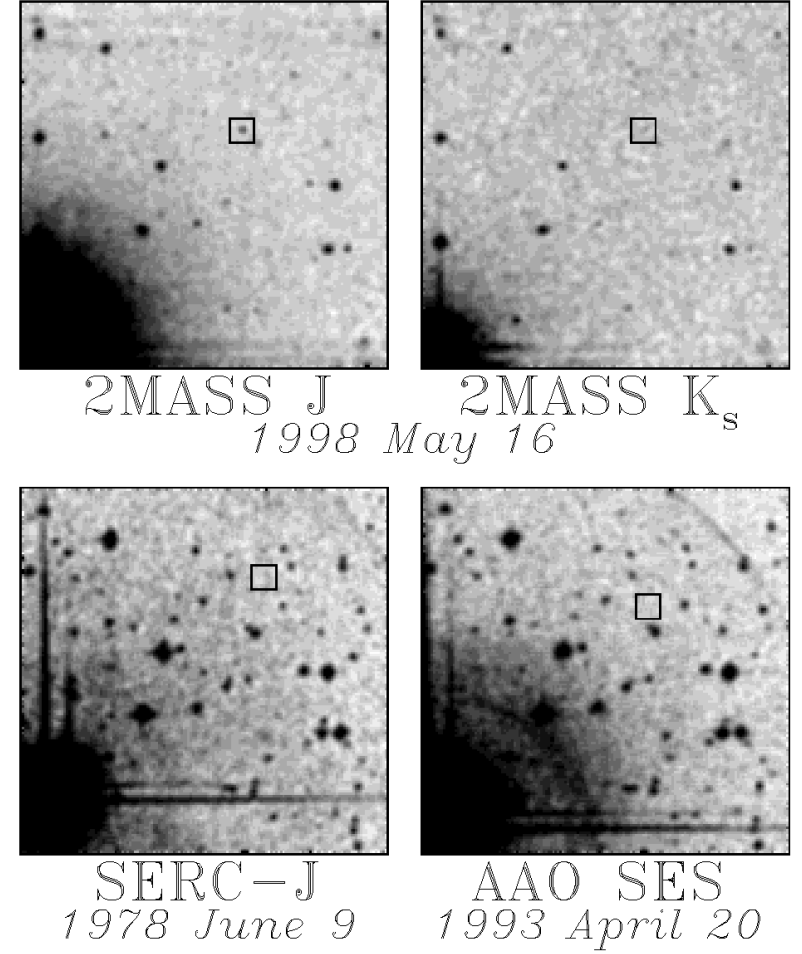

FIG. 1.-2MASS $J$ - and $K_{s}$-band images of Gl 570D, along with two optical images from SERC-J and AAO-SES at two different epochs. Each field is $5^{\prime} \times 5^{\prime}$ with north up and east to the left. The Gl 570ABC triple is seen in the lower left corner (the BC binary is unresolved). Gl 570D is indicated in the 2MASS images by a $20^{\prime \prime} \times 20^{\prime \prime}$ box, while its projected location due to motion is indicated in each optical image. No optical counterpart is seen in either of these images, limiting $R-J \gtrsim 6$.

corner. No optical counterpart is seen at either the current or projected (proper-motion) positions of Gl 570D, indicating very red optical-infrared colors. Table 1 lists 2MASS $J, H$, and $K_{s}$ magnitudes (rows 1-3) and colors (rows 4-6) for Gl 570D, as well as measurements for G196-3B and Gl 229B taken from the literature (Matthews et al. 1996) and from 2MASS data. Note that Gl 570D has blue near-infrared colors, similar to Gl 229B.

\subsection{Spectral Data}

The 1.6 and $2.2 \mu \mathrm{m}$ fundamental overtone $\mathrm{CH}_{4}$ bands were identified in G1 570D from near-infrared spectral data taken with the Ohio State Infrared Imager/Spectrometer (OSIRIS; Depoy et al. 1993) on the CTIO Blanco $4 \mathrm{~m}$ on 1999 July 27 (UT). Using OSIRIS's cross-dispersion mode, we obtained continuous $1.2-2.3 \mu \mathrm{m}$ spectra with $\lambda / \Delta \lambda \approx 1200$. The slit width was fixed at 1"2 for all observations. The object was placed on the slit by direct image centroiding, and then stepped across the slit in seven positions at $3^{\prime \prime}$ intervals (to offset fringing and detector artifacts) with $120 \mathrm{~s}$ integrations at each position. A total of $3360 \mathrm{~s}$ of integration time was acquired. Spectra were then extracted using standard IRAF reduction packages. Raw data were flat-fielded using observations of the $4 \mathrm{~m}$ illuminated dome spot and software generously supplied by R. Blum at CTIO. Object spectra were extracted using a template from the A1 V standard star HR 5696 (Hoffleit \& Jaschek 1982). Wavelength calibration was computed from $\mathrm{OH}$ sky lines. Finally, telluric corrections and relative flux calibration were done using the extracted standard spectrum.
TABLE 1

Properties of Confirmed Companion Brown Dwarfs

\begin{tabular}{|c|c|c|c|}
\hline Property & G196-3B ${ }^{a}$ & Gl 229B ${ }^{b}$ & Gl 570D ${ }^{\mathrm{c}}$ \\
\hline 1. $J$ & $14.90 \pm 0.05$ & $14.33 \pm 0.05$ & $15.33 \pm 0.05$ \\
\hline 2. $H$ & $13.67 \pm 0.07$ & $14.35 \pm 0.05$ & $15.28 \pm 0.09$ \\
\hline 3. $K_{s} \ldots$ & $12.81 \pm 0.13$ & $14.42 \pm 0.05$ & $15.27 \pm 0.17$ \\
\hline 4. $J-H$ & $1.23 \pm 0.09$ & $-0.02 \pm 0.07$ & $0.05 \pm 0.10$ \\
\hline 5. $H-K_{s}$ & $0.86 \pm 0.15$ & $-0.07 \pm 0.07$ & $0.01 \pm 0.19$ \\
\hline 6. $J-K_{s} \ldots \ldots$ & $2.09 \pm 0.14$ & $-0.09 \pm 0.07$ & $0.06 \pm 0.17$ \\
\hline 7. $\rho(\operatorname{arcsec}) \ldots$ & 16.2 & $7.8 \pm 0.1$ & $\begin{array}{l}258.3 \pm 0.4 \\
234.1 \pm 0.4^{\mathrm{d}}\end{array}$ \\
\hline 8. P.A. (deg) & 210 & 163 & $316,317^{\mathrm{d}}$ \\
\hline 9. $\rho(\mathrm{AU}) \ldots$ & $340 \pm 100$ & $44.9 \pm 0.6$ & $\begin{array}{l}1525 \pm 15 \\
1385 \pm 15^{\mathrm{d}}\end{array}$ \\
\hline 10. Distance modulus ${ }^{\mathrm{e}} \ldots .$. & $1.6 \pm 0.6$ & $-1.19 \pm 0.07$ & $-1.14 \pm 0.05$ \\
\hline $11 . M_{J} \ldots \ldots \ldots \ldots \ldots \ldots$ & $13.3 \pm 0.6$ & $15.52 \pm 0.06$ & $16.47 \pm 0.07$ \\
\hline 12. $\log \left(L / L_{\odot}\right) \ldots \ldots \ldots \ldots \ldots$ & $-3.8_{-0.3}^{+0.2}$ & $-5.18 \pm 0.04$ & $-5.56 \pm 0.05^{i}$ \\
\hline 13. Age (Gyr) ............ & $0.02-0.3$ & $0.5-1.0$ & $2-10$ \\
\hline 14. $T_{\text {eff }}(\mathrm{K}) \ldots \ldots$ & $1800 \pm 200$ & $960 \pm 70$ & $750 \pm 50$ \\
\hline 15. $M\left(M_{\text {Jup }}\right) \ldots$ & $25_{-10}^{+15}$ & $43 \pm 12$ & $50 \pm 20^{\mathrm{g}}$ \\
\hline
\end{tabular}

${ }^{\text {a }}$ Photometry from 2MASS; remaining data from Rebolo et al. 1998.

${ }^{\mathrm{b}}$ Data from Nakajima et al. 1995, Marley et al. 1996, and Leggett et al. 1999

${ }^{\mathrm{c}}$ Data for epoch 1998 May 16 (UT).

${ }^{\mathrm{d}}$ Values given are for A-D and BC-D, respectively.

e Data for Gl 229A and Gl 570A from Hipparcos (Perryman et al. 1997).

${ }^{\mathrm{f}}$ Assuming $J$-band bolometric correction of $2.19 \pm 0.10$ from Leggett et al. 1999.

${ }^{g}$ Derived from evolutionary models by Burrows et al. 1997.

The near-infrared spectrum of $\mathrm{Gl} 570 \mathrm{D}$ is shown in Figure 2, along with data for the SDSS T dwarf SDSSp J162414.37+002915.6 (hereafter SDSS1624+00; Strauss et al. 1999), obtained on the same night. Both spectra are normalized at $1.55 \mu \mathrm{m}$, with SDSS1624+00 offset vertically by a constant. Gl 229B spectral data from Geballe et al. (1996), also normalized at $1.55 \mu \mathrm{m}$, are overlaid on both for comparison (dark line). The 1.6 and $2.2 \mu \mathrm{m} \mathrm{CH}_{4}$ bands are present in all three brown dwarfs, as well as combined $\mathrm{H}_{2} \mathrm{O}$ and $\mathrm{CH}_{4}$ absorption from 1.3 to $1.5 \mu \mathrm{m}$. Suppression of flux around $2.1 \mu \mathrm{m}$ is likely due to increased $\mathrm{H}_{2}$ absorption in the low-temperature atmospheres (Lenzuni, Chernoff, \& Salpeter 1991).

There is a striking similarity in the spectral morphology of these objects; however, the overlaid spectrum of Gl 229B may indicate some subtle differences. There appears to be a slight enhancement in flux (relative to Gl 229B) in SDSS1624+00 at the blue edge of the $1.3 \mu \mathrm{m}$ absorption feature and at the base of the $1.6 \mu \mathrm{m} \mathrm{CH}_{4}$ absorption band. Conversely, the spectrum of Gl 570D does not show these features and in fact appears slightly deficient at the $1.5 \mu \mathrm{m} \mathrm{H}_{2} \mathrm{O}-\mathrm{CH}_{4}$ wing and the $2.1 \mu \mathrm{m}$ flux peak. We might expect such variations if SDSS1624+00 is warmer than Gl 229B and G1 570D cooler, as $\mathrm{CH}_{4}$ bands at 1.4 and $1.6 \mu \mathrm{m}$ should deepen as the observed temperature decreases, since the conversion of $\mathrm{CO}$ to $\mathrm{CH}_{4}$ will occur at greater optical depth (Burrows \& Sharp 1999). Similarly, there should be increased $\mathrm{H}_{2}$ absorption in the $K$-band toward lower temperatures (Burgasser et al. 1999). While metallicity and mixing effects may complicate these simple arguments, the warmer temperature of SDSS1624+00 is supported by recent detections of $\mathrm{FeH}$ and $\mathrm{CrH}$ bands in its optical spectrum (Liebert et al. 2000) which are disappearing in the latest L dwarfs (Kirkpatrick et al. 1999b), as well as shallower $\mathrm{H}_{2} \mathrm{O}$ and $\mathrm{CH}_{4}$ bands in the near-infrared as compared to Gl 229B (Nakajima et al. 2000). The coolness of Gl 570D, based on its association with G1 570ABC, is discussed below. 


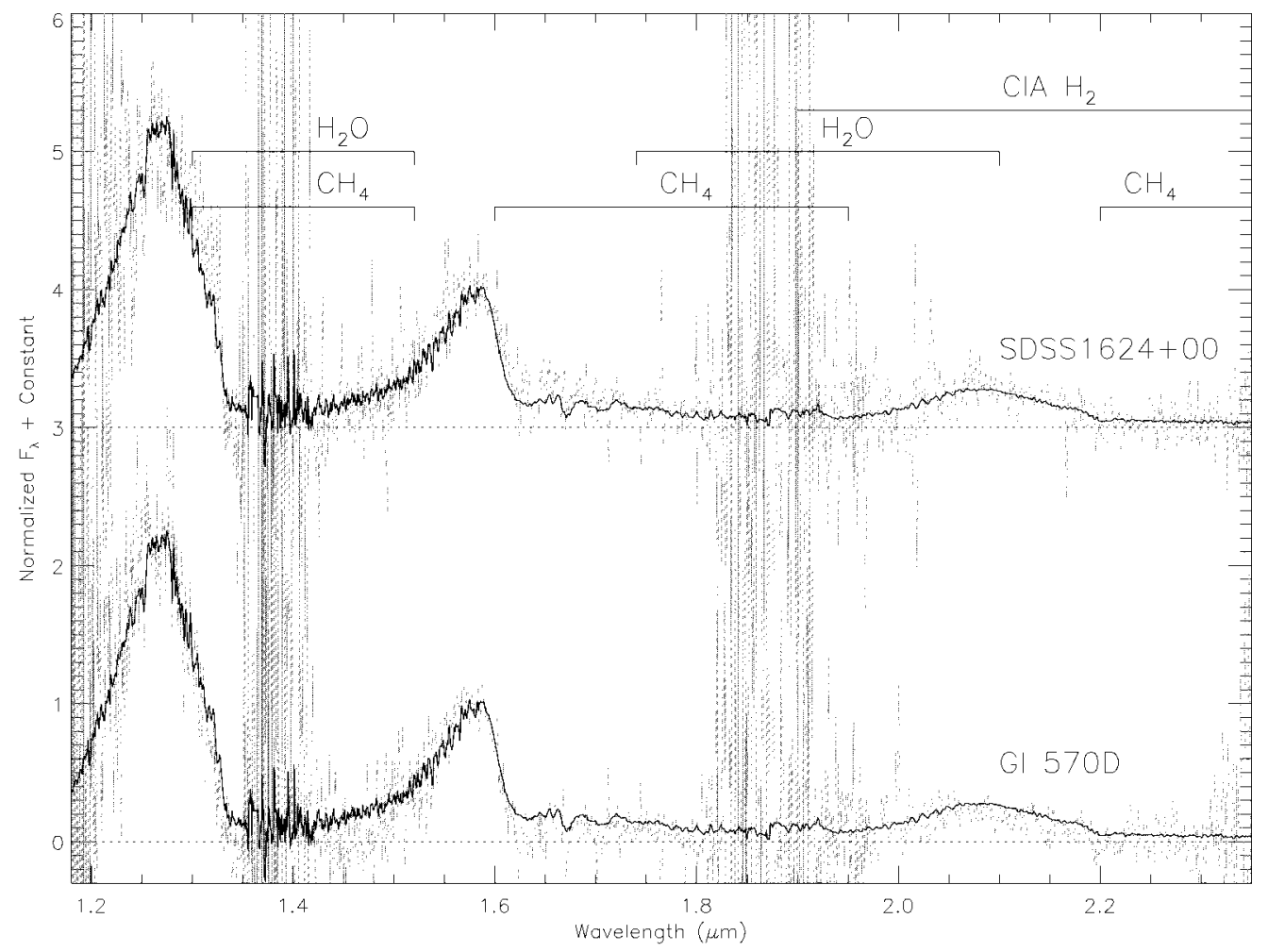

FIG. 2.-Near-infrared spectral data for Gl 570D (bottom) and SDSS1624+00 (top). Both are normalized at $1.55 \mu \mathrm{m}$ with spectral data for Gl 229B from Geballe et al. (1996) overlaid for comparison (dark line). An integral offset (3.0) has been added to the spectra in the top panel to separate them vertically from the others, and zero levels are indicated by dashed lines. The data are strikingly similar: $\mathrm{CH}_{4}$ absorption features at $1.3,1.6$, and $2.2 \mu \mathrm{m}$ are clearly seen in all three objects, as are broadened $\mathrm{H}_{2} \mathrm{O}$ absorption bands at 1.3 and $1.9 \mu \mathrm{m} . \mathrm{H}_{2}$ collision-induced absorption (CIA) longward of about $1.9 \mu \mathrm{m}$ is probably also present. Despite the strong similarities, there are some variations in flux near the 1.3 and $1.5 \mu \mathrm{m} \mathrm{H}_{2} \mathrm{O}-\mathrm{CH}_{4}$ absorption wings, the $1.6 \mu \mathrm{m} \mathrm{CH}$ absorption band, and the $2.1 \mu \mathrm{m}$ flux peak, all of which could be attributable to temperature differences between these objects.

\subsection{Association with $G l 570 A B C$}

The proximity of the bright Gl 570ABC triple led us to suspect possible association for this 2MASS object. Fortunately, the system has a relatively high proper motion of 2 .012 $\pm 0.002 \mathrm{yr}^{-1}$ (Perryman et al. 1997). In addition, multiple sampling and the 2MASS position reconstruction strategy results in a higher astrometric accuracy ${ }^{13}\left(\sim 00^{\prime \prime} 3\right)$ than the raw pixel scale of the 2MASS detectors ( $\left.2^{\prime \prime}\right)$, sufficient to measure the motion of this system on a $1 \mathrm{yr}$ timescale. The original 2MASS scan of the Gl 570D field was taken on 1998 May 16 (UT); a second scan was obtained on 1999 July 29 (UT). Table 2 summarizes the resulting astrometric data, indicating that all components have a common sky motion of $2.3 \pm 0$ 0".4 at position angle $155^{\circ} \pm 8^{\circ}$. The mean motion of all other correlated sources in the same 2MASS scan as Gl 570D with $J<15.8$

\footnotetext{
${ }^{13}$ R. M. Cutri et al. 1999, Explanatory Supplement to the 2MASS Spring 1999 Incremental Data Release (http://www.ipac.caltech.edu/2mass/releases/ spr99/doc/explsup.html).
}

$\left(\approx 2000\right.$ sources) is $00^{\prime \prime} 0 \pm 00^{\prime \prime} 2$ in right ascension and $00^{\prime \prime} 2 \pm$ $0{ }^{\prime \prime} 1$ in declination. This statistically significant common proper motion confirms companionship. Gl 570D lies 258".3 \pm 0".4 from the $\mathrm{K} 4 \mathrm{~V}$ primary, a projected physical separation of $1525 \pm 15 \mathrm{AU}$. Note that this is an order of magnitude larger than the A-BC separation (24."7 \pm 0 ".4) and over 3 orders of magnitude larger than the B-C separation of $0{ }^{\prime \prime} 1507 \pm$ 0"0007 (Forveille et al. 1999). The separation of Gl 570D is compared to those of G196-3B and Gl 229B in Table 1 (rows 7-9).

The dynamic stability of this system can be addressed using the results of Eggleton \& Kiseleva (1995) with the separations ${ }^{14}$ listed in Table 2 and masses of $0.7 M_{\odot}$ for Gl 570A (estimated from the measured mass of the M0 Ve eclipsing binary YY Gem; Bopp 1974), 1.0 $M_{\odot}$ for combined Gl 570BC (directly measured by Forveille et al. 1999), and $0.05 M_{\odot}$ for Gl 570D

\footnotetext{
${ }^{14}$ We assume face-on projection and negligible eccentricity in this orderof-magnitude analysis.
}

TABLE 2

2MASS Astrometry For Gl 570ABCD

\begin{tabular}{|c|c|c|c|c|c|c|}
\hline \multirow[b]{2}{*}{ COMPONENT } & \multicolumn{2}{|c|}{1998 MAY 16 (UT) } & \multicolumn{2}{|c|}{1999 JULY 29 (UT) } & \multicolumn{2}{|c|}{ DIFFERENCE } \\
\hline & R.A. & Decl. & R.A. & Decl. & $(\operatorname{arcsec})$ & (deg) \\
\hline A... & 145727.87 & -212452.72 & 145727.93 & -212454.87 & $2.3 \pm 0.4$ & $159 \pm 8$ \\
\hline $\mathrm{BC}$ & 145726.42 & -212438.54 & 145726.49 & -212440.77 & $2.4 \pm 0.4$ & $156 \pm 7$ \\
\hline D... & 145714.96 & -212147.79 & $1457 \quad 15.04$ & -212149.82 & $2.3 \pm 0.4$ & $151 \pm 8$ \\
\hline
\end{tabular}

Note. - Units of right ascension are hours, minutes, and seconds, and units of declination are degrees, arcminutes, and arcseconds. All coordinates are equinox J2000.0. 
(estimated, as discussed below). We find that the system is dynamically stable for eccentricities less than about 0.6. A more rigorous analysis using measured orbital parameters is restricted by the roughly $40,000 \mathrm{yr}$ period of Gl 570D around the Gl 570ABC barycenter.

\section{ESTIMATES OF THE PHYSICAL PROPERTIES OF G1 570D}

The distance moduli and absolute $J$ magnitudes for the three brown dwarf companions G196-3B, Gl 229B, and Gl 570D, based on the distances to their respective primaries, are listed in Table 1 (rows 10-11). Gl 570D is nearly a magnitude fainter than Gl 229B at all three near-infrared bands. If we assume a G1 229B $J$-band bolometric correction of $2.19 \pm 0.10$ (Leggett et al. 1999) and a radius of $(7.0 \pm 0.5) \times 10^{9} \mathrm{~cm} \approx 1$ Jupiter radius (Burrows \& Liebert 1993), we then derive $L=(2.8 \pm$ $0.3) \times 10^{-6} L_{\odot}$ and $T_{\text {eff }}=750 \pm 50 \mathrm{~K}$, roughly $200 \mathrm{~K}$ cooler than Gl 229B, making Gl 570D the least luminous and coolest brown dwarf thus far detected. More accurate determinations of the effective temperature and mass of Gl 570D can be made using brown dwarf evolutionary models, but only if we can constrain its age $(\tau)$. The proximity of Gl 570ABC has permitted detailed studies of kinematic properties, activity, and high-energy emission (UV and X-ray), leading to various age estimates for the system (Leggett 1992; Poveda et al. 1993; Fleming, Schmitt, \& Giampapa 1995). There is a general consensus among these authors that this system is older than 2 Gyr, which is supported by the lack of activity in the close BC binary (Reid, Hawley, \& Gizis 1995). The solar-like metallicity of Gl 570ABC (Forveille et al. 1999) and the system's total space motion of $\approx 60 \mathrm{~km} \mathrm{~s}^{-1}$ (Leggett 1992) constrains formation to the Galactic disk, which establishes a rough upper limit of about 10 Gyr. Using the evolutionary models of Burrows et al. (1997) and adopting $\log \left(L / L_{\odot}\right)=-5.56 \pm 0.05$ and $\tau=6 \pm 4 \mathrm{Gyr}$, we derive values of $T_{\text {eff }}=790 \pm 40 \mathrm{~K}$ and
$M=50 \pm 20 M_{\text {Jup }}^{15}$ (Table 1, rows 14-15). The effective temperature is consistent with the brightness estimate above and is significantly lower than those of G196-3B and Gl 229B. Perhaps most interesting is that, despite having the lowest $T_{\text {eff }}$, Gl 570D could possibly be the most massive of these three brown dwarfs. This accentuates the difficulty of basing comparisons of brown dwarfs on brightness and/or temperature alone and the importance of age determinations in deriving the physical properties of cool brown dwarfs. More accurate estimates of this object's properties require spectral modeling and additional broadband photometry and will be addressed in a future paper.

A. J. B. acknowledges Robert Blum and Ron Probst for their guidance at the telescope and in the reduction process, the capable assistance of CTIO telescope operators Mauricio Fernandez and Alberto Zúniga, useful discussions with Mark Marley, helpful comments from the anonymous referee, and Daniel Durand for dealing with high volumes of image requests on the CADC DSS server. We thank the 2MASS staff and scientists for their efforts in creating a truly incredible astronomical resource. DSS images were obtained from the Canadian Astronomy Data Centre, which is operated by the Herzberg Institute of Astrophysics, National Research Council of Canada. A. J. B., J. D. K., I. N. R., and J. L. acknowledge funding through a NASA/JPL grant to 2MASS Core Project science. A. J. B., J. D. K., R. M. C., and C. A. B. acknowledge the support of the Jet Propulsion Laboratory, California Institute of Technology, which is operated under contract with the National Aeronautics and Space Administration. This publication makes use of data from the Two Micron All-Sky Survey, which is a joint project of the University of Massachusetts and the Infrared Processing and Analysis Center, funded by the National Aeronautics and Space Administration and the National Science Foundation.

$$
{ }^{15} 1 M_{\text {Jup }}=1.9 \times 10^{33} \mathrm{~g}=0.0095 M_{\odot} .
$$

\section{REFERENCES}

Becklin, E. E., \& Zuckerman, B. 1989, Nature, 336, 656

Bopp, B. W. 1974, ApJ, 193, 389

Burgasser, A. J., et al. 1999, ApJ, 522, L65

- 1998, BAAS, 30, 1393

Burrows, A., \& Liebert, J. 1993, Rev. Mod. Phys., 65, 301

Burrows, A., \& Sharp, C. M. 1999, ApJ, 512, 843

Burrows, A., et al. 1997, ApJ, 491, 856

Depoy, D. L., Atwood, B., Byard, P. L., Frogel, J., \& O'Brien, T. P. 1993, Proc. SPIE, 1946, 667

Duquennoy, A., \& Mayor, M. 1988, A\&A, 200, 135

Eggleton, P., \& Kiseleva, L. 1995, ApJ, 455, 640

Epchtein, N., et al. 1997, Messenger, 87, 27

Fischer, D. A., \& Marcy, G. W. 1992, ApJ, 396, 178

Fleming, T. A., Schmitt, J. H. M. M., \& Giampapa, M. S. 1995, ApJ, 450, 401

Forveille, T., et al. 1999, A\&A, 351, 619

Geballe, T. R., Kulkarni, S. R., Woodward, C. E., \& Sloan, G. C. 1996, ApJ, 467, L101

Gunn, J. E., \& Weinberg, D. H. 1995, in Wide-Field Spectroscopy and the Distant Universe, ed. S. Maddox \& A. Aragón-Salamanca (Singapore: World Scientific), 3

Hoffleit, D., \& Jaschek, C. 1982, The Bright Star Catalogue (4th ed.; New Haven: Yale Univ. Obs.)

Kirkpatrick, J. D., Allard, F., Bida, T., Zuckerman, B., Becklin, E. E., Chabrier, G., \& Baraffe, I. 1999a, ApJ, 519, 834

Kirkpatrick, J. D., et al. 1999b, ApJ, 519, 802

Leggett, S. K. 1992, ApJS, 82, 351

Leggett, S. K., Toomey, D. W., Geballe, T. R., \& Brown, R. H. 1999, ApJ, 517, L139

Lenzuni, P., Chernoff, D. F., \& Salpeter, E. E. 1991, ApJS, 76, 759
Liebert, J., Reid, I. N., Burrows, A., Burgasser, A. J., Kirkpatrick, J. D., \& Gizis, J. E. 2000, ApJ, submitted

Mariotti, J.-M., Perrier, C., Duquennoy, A., \& Duhoux, P. 1990, A\&A, 230, 77

Marley, M. S., Saumon, D., Guillot, T., Freedman, R. S., Hubbard, W. B., Burrows, A., \& Lunine, J. I. 1996, Science, 272, 1919

Matthews, K., Nakajima, T., Kulkarni, S. R., \& Oppenheimer, B. R. 1996, AJ, 112,1678

Monet, D., et al. 1998, USNO-A2.0 Catalog (Flagstaff: USNO)

Morgan, D. H., Tritton, S. B., Savage, A., Hartley, M., \& Cannon, R. D. 1992, in Digitised Optical Sky Surveys, ed. H. T. MacGillivray \& E. B. Thomson (Dordrecht: Kluwer), 11

Nakajima, T., Oppenheimer, B. R., Kulkarni, S. R., Golimowski, D. A., Matthews, K., \& Durrance, S. T. 1995, Nature, 378, 463

Nakajima, T., et al. 2000, PASJ, in press

Oppenheimer, B. R. 1999, Ph.D. thesis, Caltech

Perryman, M. A. C., et al. 1997, A\&A, 323, L49

Poveda, A., Herrera, M. A., Allen, C., Cordero, G., \& Lavalley, C. 1994, Rev. Mexicana Astron. Astrofis., 28, 43

Rebolo, R., Zapatero Osorio, M. R., Madruga, S., Bejar, V. J. S., Arribas, S., \& Licandro, J. 1998, Science, 282, 1309

Reid, I. N., Hawley, S. L., \& Gizis, J. E. 1995, AJ, 110, 1838

Skrutskie, M. F., et al. 1997, in The Impact of Large-Scale Near-IR Sky Surveys, ed. F. Garzon (Dordrecht: Kluwer), 25

Strauss, M. A., et al. 1999, ApJ, 522, L61

Sykes, M. V., Cutri, R. M., Fowler, J. W., Tholen, D. J., Skrutskie, M. F., Price, S., \& Tedesco, E. F. 1999, AAS, DPS Meeting 31, 59.15

Veeder, G. J., Matson, D. L., Owensby, P. D., Gradie, J. C., Bell, J. F., \& Tedesco, E. F. 1995, Icarus, 114, 186 